\title{
Embryonic death and the creation of human embryonic stem cells
}

\author{
Donald W. Landry 1 and Howard A. Zucker ${ }^{2}$ \\ 1Department of Medicine and 2Department of Pediatrics, Columbia University College of Physicians and Surgeons, New York, New York, USA.
}

\begin{abstract}
The creation of human embryonic stem cells through the destruction of a human embryo pits the value of a potential therapeutic tool against that of an early human life. This contest of values has resulted in a polarized debate that neglects areas of common interest and perspective. We suggest that a common ground for pursuing research on human embryonic stem cells can be found by reconsidering the death of the human embryo and by applying to this research the ethical norms of essential organ donation.
\end{abstract}

\section{Introduction}

Human embryonic stem cells may or may not possess the innate capacity to provide unique treatments for human disease. But at least for now, live human embryos must be destroyed in the process of creating stem cells. The questionable ethics of such destruction led the US Congress to enact a broad ban on federal funding for human embryonic stem cell research. In 2001, President Bush modified this ban by removing restrictions for work with existing lines, but he continued prohibition of funding for the creation of new lines. However, existing lines may prove inadequate, and the eventual need for new embryonic lines cannot be discounted. Also, research on nonhuman embryonic cells is often not easily translated to humans; thus active research on human embryonic cells is desirable. Nonetheless, the duty to heal the sick cannot override the moral imperative to treat human beings as subjects and not objects. We propose herein a paradigm for research involving embryos that protects nascent human life, is consistent with current federal policy, and yet advances the interests of biomedical science and therapeutic innovation.

Precisely when the life of a human begins remains for some a complicated question, but a general consensus has been achieved on when life ends: life ends when the criteria for brain death are met. However, the criteria for determining the death of the developing human before the onset of neural development have not been formulated. We believe that when the condition of developing human life at the stage of a few-celled embryo is reconsidered, a significant fraction of embryos generated for in vitro fertilization (IVF), heretofore misclassified as nonviable, will be found to be organismically dead. If this is so, the ethical framework currently used for obtaining essential organs for transplantation from deceased adults and children could be extended to cover obtaining stem cells from dead human embryos.

\section{Background on embryonic stem cells}

Methods for preparing embryonic stem cells were originally developed in mice in 1981, but human lines were successfully isolated only recently (1); the time lag underscores the problem posed by the interspecific transfer of such technology. In order for human cells to be obtained, donated embryos or embryos generated from

Nonstandard abbreviations used: ICM, inner cell mass; IVF, in vitro fertilization. Conflict of interest: The authors have declared that no conflict of interest exists.

Citation for this article: J. Clin. Invest. 114:1184-1186 (2004).

doi:10.1172/JCI200423065 donated eggs and sperm are grown in culture to the blastocyst stage (Figure 1). The blastocyst forms at approximately 4 or 5 days after fertilization and contains from 64 to several hundred cells organized in an outer shell, the trophectoderm, and a collection of polarized inner cells termed the inner cell mass (ICM). The ICM is the locus of pluripotent cells destined to yield all the tissues of the developed organism. In the process of obtaining embryonic stem cells, the trophectoderm is removed by immunosurgery, and the ICM is disaggregated and plated on feeder cells. The resulting cell colonies are mechanically isolated and replated until homogenous colonies are obtained (2). The colonies are then screened for the presence of stem cells. The desired cells are clonogenic and capable of dividing symmetrically for unlimited self-renewal. These cells are also capable of dividing asymmetrically to yield one cell identical to the parent and one able to develop into diverse cell types. Once established, human embryonic stem cell lines can persist stably, apparently for years. At present there is no source of new embryonic human stem cell lines that does not involve the destruction of human embryos.

\section{Definition of death and the ethics of essential organ donation}

The evolution of the definition of death for developed human beings can illuminate the consideration of death for developing humans. Prior to the 1960s, the legal definition of death focused on irreversible cessation of cardiopulmonary function (3). Advances in respiratory technology that could maintain a body indefinitely highlighted the inadequacy of this definition and an ad hoc committee at Harvard Medical School in 1968 formulated a new criterion for determining whether death had occurred: the state of irreversible coma (4). US Federal and State courts treat the determination of human death as a specific question of fact to be decided case by case rather than a general question of law (4). Thus implementing the 1968 criterion was not contingent upon statutory enactment, but to avoid confusion, laws were passed. The first legislative attempt in 1970 to frame "[a]n Act relating to and defining death" was criticized on the grounds that it provided for two separate definitions for death and that the definition used in individual cases was contingent only upon the presence or absence of a desire to harvest organs for transplantation (5). Capron and Kass in 1972 proposed that there be a single concept of death but that, depending on the absence or presence of the machinery of life support that made recognizing it uncertain, either irreversible cessation of spontaneous circulatory and respiratory functions or irreversible cessation 


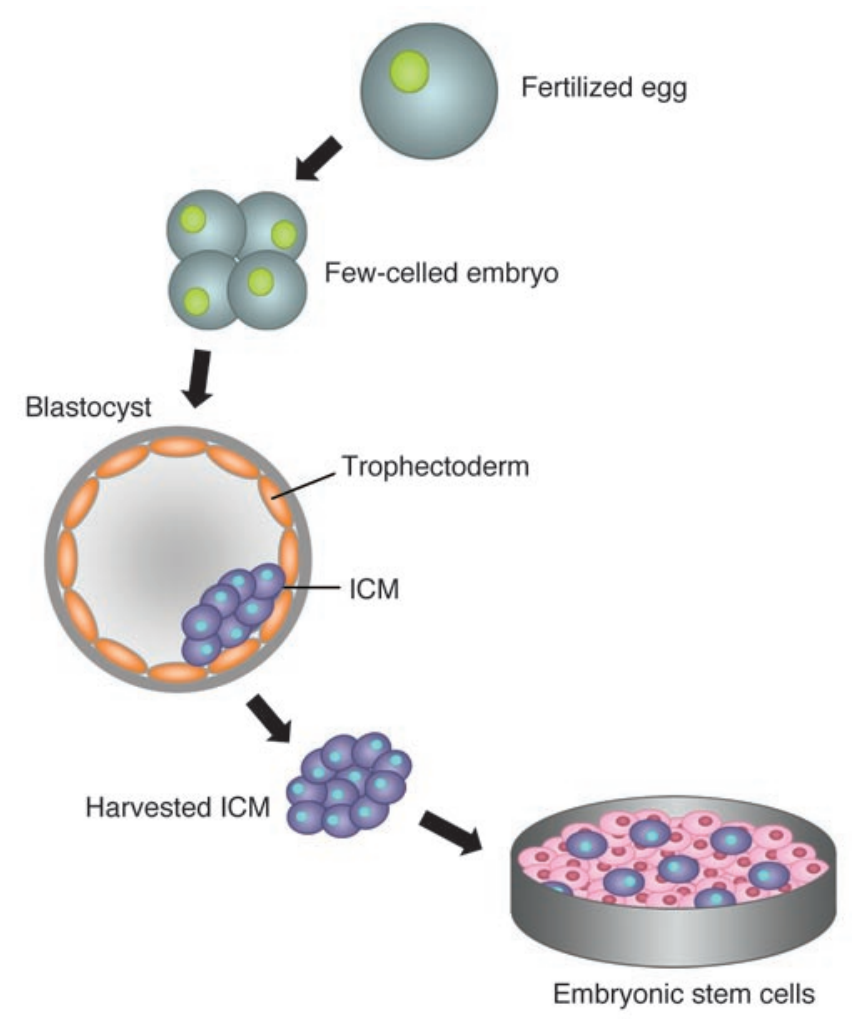

Figure 1

Human embryonic stem cells. The steps from fertilized egg obtained by IVF to embryonic stem cells are depicted. A few-celled embryo gives rise to the blastocyst, a structure comprised of an outer cell layer, the trophectoderm, and the ICM. The ICM is harvested and plated on feeder cells, then replated (not shown) to yield a population of embryonic stem cells.

of brain functions could be used to determine whether the person was in fact dead (6). Medical, legal, and ethical opinion coalesced around the concept of brain death, and the Capron-Kass proposal combined with the 1975 American Bar Association (ABA) Definition of Death Act ultimately led to the 1981 Uniform Determination of Death Act (UDDA) (7), widely adopted by individual states. It is "the law's determination that brain death is the legal equivalent of death because ... the capacity for life is irretrievably lost when the entire brain, including the brain stem, has ceased functioning" (5). Legal challenges to this definition of death have been unsuccessful $(5,8)$. More recently, in 1995, the definitions regarding clinical and confirmatory test requirements for brain death were reexamined by the American Academy of Neurology (9), and the criteria set forth by the Harvard committee were reaffirmed. Thus, at the time of brain death, the removal of vital organs for transplant is legally and ethically permitted. As stated by the Omnibus Reconciliation Act of 1986 (10), after brain death criteria have been met, the next of kin may be approached regarding organ donation.

A fully developed human being is considered organismically dead - even as the cells of the various organs remain alive - if the function of the brain is irretrievably lost. The transition from life to organismic death is determined to have occurred if the criteria for brain death are met. But how can one determine organismic death at the earliest stages of a human life, at a time still weeks from the inception of the central nervous system?

\section{The concept of organismic death for the human embryo}

For a developed human organism, brain death marks the irreversible loss of the capacity for all ongoing and integrated organic functioning. We propose that the defining capacity of a 4- or 8-cell human embryo is continued and integrated cellular division, growth, and differentiation. We further propose that an embryo that has irreversibly lost this capacity, even as its individual cells are alive, is properly considered organismically dead. Even at its earliest stages, the life of the developing organism is more than the sum of the lives of its constituent cells. Each cell is involved in directing the gene expression and differentiation of other cells and, thereby, the growth and development of the whole. Just as the nervous system integrates the activities of the tissues and organs of the developed organism, so a system of chemical communication and surface recognition integrates the cells of the developing organism. The determinants of growth and differentiation, such as Oct4, are now being elucidated (11). If an embryo is deprived of the necessary internal signals, irreversible arrest of cleavage can result. Objective criteria for diagnosing death require straightforward methods for determining irreversible arrest. The determination of irreversibility could be inductive, based on observing the natural history of cleavage arrest in large numbers of embryos, or deductive, based on elucidating biochemical defects that preclude further growth and development - or both. Studies of IVF embryos suggest that irreversibly arrested embryos could contain cells with normal developmental potential.

\section{The prospect for producing normal cells from dead embryos}

Approximately $60 \%$ of IVF embryos fail to meet criteria for viability and are rejected for uterine transfer. Nonviability, defined as the incapacity to develop to live birth, differs from organismic death; all dead embryos are, of course, nonviable, but most nonviable embryos are not yet dead. Morphological criteria for nonviability include abnormal cleavage, loss of cells, and loss of cytoplasm. But it is the functional criterion - failure to cleave at 24 hours - that, while not by itself proof of irreversibility, likely correlates. Cleavage arrest most often reflects severe genetic abnormalities but - and this is the crucial point - not all cells of arrested embryos need be abnormal for arrest to occur. In a study by Laverge et al., out of 166 frozen embryos thawed for further growth, 78 embryos remained arrested at 24 hours after thawing, and 71 showed no sign of further cleavage at 48 hours (12). Fluorescence in situ hybridization with probes for chromosomes $\mathrm{X}, \mathrm{Y}$, and 1 was performed on all blastomeres of 63 arrested embryos, and $80 \%$ showed genetic abnormalities. Aneuploidy was common, but defects were both meiotic and mitotic in origin, and mosaic embryos containing at least one normal diploid blastomere were identified. Voullaire et al. performed comparative genomic hybridization on 65 blastomeres from 12 unselected embryos (13). Frozen human embryos, scheduled to be discarded after 5 years of storage, were thawed, observed in culture for 24 hours for cleavage, allowed to expire at room temperature, and then disaggregated for analysis. Marked aneuploidy was noted, but once again mosaic embryos (5 out of 12) with at least one normal blastomere were observed. Thus an unexpectedly high level of mosaicism due to postzygotic nondisjunction, i.e., defective segregation of chromosomes in a single cell after the first cell division, could provide a potential source of normal blastomeres from organismically dead embryos. 
Several studies provide insights into the possible developmental potential of blastomeres derived from dead human embryos. In a study in frogs by Byrne et al., tadpole embryos that were derived by nuclear transplantation (i.e., cloned) rarely developed into tadpoles (less than $1 \%$ ) because a majority underwent irreversible arrest and began to decompose within 24 hours (14). However, if the cells of an arrested blastocyst were disaggregated and injected into normal tadpole blastocysts, those extracted cells frequently resumed division $(25 \%)$ and were stably incorporated into differentiated tissues. Further, in ethically troubling experiments by Alikani and Willadsen, 107 IVF embryos designated as "nonviable" by the authors were disaggregated, and 247 intact cells were isolated and combined in 36 aggregates (15). Among these aggregates, $33 \%$ formed normally organized blastocysts with defined inner cell masses. These experiments indicate that it is likely that blastomeres from organismically dead embryos can maintain their development potential. Detailed studies will be needed to assess the presence of any chromosomal damage in these blastomeres that might affect their long-term function in differentiated tissues.

Damage that must be excluded in the surviving blastomeres includes subtle chromosomal fragmentation, methylation defects, and telomerase anomalies. A natural history study to define human embryonic death could be organized as follows: previously frozen early embryos that have failed to divide within 24 hours of thawing and are no longer wanted for their original reproductive purpose are observed every few hours for several additional 24-hour periods. After observing several hundred embryos, the time beyond which no arrested embryo resumes division can be determined. One can reasonably conclude that embryos that have not divided by this period after thawing will not divide at any later time, i.e., they are organismically dead. Embryos declared dead could then be characterized for secreted or cell surface markers or spectroscopic signatures that correlate with the arrest of cell division. These markers and signatures could then be tested for their predictive value. In this manner the criteria for determining the death of a human embryo could be refined.

\section{Conclusion}

A reality of human embryonic life whether in utero or in vitro is a high incidence of death in the first few days after fertilization. We propose that an irreversible arrest of cell division rather than the death of each and every cell is the appropriate measure of organis- mic death for the embryo. Criteria for determining irreversibility are lacking, but the approach is clear; natural history studies of cleavage arrest will provide an initial definition that can be refined as elucidation of the mechanisms regulating growth and arrest yield biochemical markers for irreversibility. Based on our analysis, we believe that many embryos generated for IVF are dead at the organismic level and yet, due to mosaicism, are likely donors of normal blastomeres. Although the signals required for transforming blastomeres into stem cells - without recourse to the formation and destruction of human blastocysts - remain to be elucidated, relatively unfettered access to human blastomeres would advance this research program.

The legal and administrative process that led to criteria for establishing the death of the developed human provides insights into the comparable process necessary for determining death during embryonic development. If the donation of embryonic cells from organismically dead embryos is considered as analogous to the donation of essential organs from cadavers, the result could be widespread acceptance of the use of such cells for research and development. Species specificity renders research on human cells all the more important, and access to blastomeres from organismically dead embryos would facilitate innovation. As an example, human blastomeres may be suitable for somatic cell cloning with the potential to circumvent the destruction of live human embryos that characterizes the current protocols for "therapeutic cloning." The harvesting of organs from executed prisoners or aborted fetuses is criticized for encouraging executions and abortions. In contrast, the harvesting of cells from organismically dead embryos would not seem to significantly encourage the practice of in vitro fertilization for infertility, a practice in which death is undesired rather than the primary objective. In sum, application of the ethical framework for essential organ donation to the harvesting of human embryonic cells from dead embryos could provide a common ground in which the imperative to safeguard human dignity and the drive for biomedical research are not in conflict.

\section{Acknowledgments}

We thank Virginia Papaioannou for helpful discussions.

Address correspondence to: Donald W. Landry, Columbia University, P\&S Building 10th floor, Room 445, 630 West 168th Street, New York, New York 10032, USA. Phone: (212) 305-2436; Fax: (212) 305-3475; E-mail: dwl1@columbia.edu.
1. Thomson, J.A., et al. 1998. Embryonic stem cell lines derived from human blastocysts. Science. 282:1145-1147.

2. Rosenthal, N. 2003. Prometheus's vulture and the stem-cell promise. N. Engl. J. Med. 349:267-274.

3. Black, H.C. 1990. Black's law dictionary: definitions of the terms and phrases of American and English jurisprudence, ancient and modern. 6th edition. West Publishing Co. St. Paul, Minnesota, USA. 1657 pp.

4. Ad Hoc Committee of the Harvard Medical School. 1968. A definition of irreversible coma. JAMA. 205:337-340.

5. Kansas Statutes Annotated. sec. 77-202 (Supp. 1971).

6. Capron, A., and Kass, L. 1972. A statutory definition of the standards for determining human death; an appraisal and a proposal. University of Pennsylvania Law Review. 121:87.
7. President's Commission for the Study of Ethical Problems in Medicine and Biomedical and Behavioral Research. 1981. Defining death: a report on the medical, legal and ethical issues in the determination of death. The Commission: for sale by the Superintendent of Documents, US Government Printing Office. Washington, D.C., USA. 166 pp.

8. In Re Long Island Jewish Medical Center, 641 New York Supplement 2d 989 (New York Superior Court 1996)

9. Report of the Quality Standards Subcommittee of the American Academy of Neurology. 1995. Practice parameters for determining brain death in adults. Neurology. 45:1912.

10. Omnibus Reconciliation Act of 1986. 42 United States Code Sec. 1320b-8.

11. Nichols, J., et al. 1998. Formation of pluripotent stem cells in the mammalian embryo depends on the POU transcription factor Oct4. Cell. 95:379-391.

12. Laverge, H., et al. 1998. Fluorescent in-situ hybridization on human embryos showing cleavage arrest after freezing and thawing. Hum. Reprod. 13:425-429.

13. Voullaire, L., Slater, H., Williamson, R., and Wilton, L. 2000. Chromosome analysis of blastomeres from human embryos by using comparative genomic hybridization. Hum. Genet. 106:210-217.

14. Byrne, J.A., Simonsson, S., and Gurdon, J.B. 2002. From intestine to muscle: Nuclear reprogramming through defective cloned embryos. Proc. Natl. Acad. Sci. U.S. A. 99:6059-6063.

15. Alikani, M., and Willadsen, S. 2002. Human blastocysts from aggregated mononucleated cells of two or more non-viable zygote-derived embryos. Reprod. Biomed. Online. 5:56-58. 